\title{
Difficulty in Management of Advanced Pediatric Orbital Tumor
}

\author{
Authors: \\ Nandang Sudrajat*(i) \\ Delfitri Lutfi

\section{Affiliations:} \\ Department of Ophthalmology, \\ Faculty of Medicine, Universitas \\ Airlangga-RSUDDr. Soetomo \\ Surabaya, Indonesia.
}

\section{Corresponding author: Nandang Sudrajat nandang.sudrajat-2017@ \\ fk.unair.ac.id}

\section{Dates:}

Received: 17 July 2021

Revised: 15 September 2021

Accepted: 21 September 2021

Published: 01 November 2021

DOI:

https://doi.org/10.20473/ vsehj.v1i1.2021.6-9

\section{Copyright:}

(C) 2021 Author(s). Open access under Creative Commons Attribution-Share Alike 4.0 International Licence (CC-BY-SA).

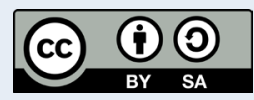

\begin{abstract}
Introduction: Tertiary care centers often manage pediatric orbital tumors, especially in advanced lesions and complex management. We report a case of a young boy with a malignant orbital tumor involving intracranial infiltration. Case presentation: A fouryear-old boy had proptosis on the left eye for two months accompanied with blurred vision, pain, and oftenly bleeds with ipsilateral nasal congestion. There was no history of trauma, eye disorders, systemic diseases, or growth disorders. The physical examination showed stable vital signs, however a weak general condition and no enlarged regional lymph nodes. Visual acuity of the left eye was no light perception. There was a mass with protrusion of the left eye, swelling of the eyelid with an irregular surface, and a tendency to bleeds. The cornea was hazy with partly scarring, so we could not evaluate the posterior segment. MRI of the head presented a malignant soft tissue mass of the left orbital region with intracranial infiltration. The patient was given adjuvant chemotherapy; however, he could not survive due to systemic complications. Conclusions: The definitive diagnosis for the orbital tumor is obtained by histopathological examination. The investigation with CT scan or MRI imaging could be considered if not possible. However, since the definitive diagnosis still not assessed, the management can be affected. It is essential to have a definitive diagnosis to provide adequate treatment for the patients. Delayed and inadequate management can make malignant orbital tumors potentially life-threatening.

Keywords: pediatric orbital tumor; advanced case; case report
\end{abstract}

\section{Introduction}

The various presentation forms of orbital neoplasms in the pediatric population are different with the same lesion in adults. Most pediatric ophthalmic tumors are benign; however, some may significantly impact vision and cause mortality. Some hereditary tumors can appear at less than one year, while others can be seen at an older age. Examination of clinical signs in orbital neoplasms must be concern with proptosis, squint, ocular motility restriction, globe displacement, decreased vision, high intraocular pressure, eyelids ecchymosis, conjunctival chemosis pseudo-hypopyon (white layer of tumor cells in the inferior anterior chamber), and pupillary afferent defect. The most common pediatric orbital tumors are rhabdomyosarcoma, neuroblastoma, and glioma. Retinoblastoma can present a similar clinical picture in the late extraocular phase. Evaluation for the metastatic disease may include imaging with orbital and neck CT or MRI, chest CT, lumbar puncture, bone scan, bilateral bone marrow aspiration, and core biopsy of the iliac crest. The primary diagnosis made by open biopsy of the primary tumor. ${ }^{[1]}$ The critical point to sound management of orbital tumors in children was the early diagnosis and treatment. Delay in the management can be life-threatening due to metastases to vital organs. This report provides an overview of patients with orbital tumors who were treated too late. It is hoped that it can provide the insight for appropriate early management.

\section{Case presentation}

A four-year-old boy came to the outpatient clinic with a protrusion in his left eye two months ago before visit. The first complaint was the left eye was more prominent than the right eye with blurred vision. The left globe position 
was gradually lowered than the right eye, painful and oftenly bleeds. There was left nasal congestion. There was no history of cat's eye appearance, other abnormalities in the left eye, trauma, seriously illness or underwent any previous surgery. The growth status of the patient was within normal limit. There was significant weight loss in the last two months.

The patient was born normally and aterm, without abnormalities during pregnancy (Figure 1A). According to the patient's parents, there was no family history of malignancy. The parents were delay for taking the patient for to get any treatment because they thought that the disease was not severe. The general condition was weak, however, the patient's vital signs are stable, with a pain scale of 3-4. There were no regional enlarged lymph nodes. The visual acuity of the left eye was no light perception. There was a protrusion of the left eye with a size of $5 \times 8 \times 3 \mathrm{~cm}$, swelling of the eyelids and venectasia. conjunctival and scleral hyperemia, irregular surface, 360-degree chemosis, fragile, and bleeds easily. There was exposed cornea with a cloudy surface and a partial leukoma. Other details of the anterior and posterior segments could not be evaluated (Figure 1B). The right eye was within the standard normal limit.

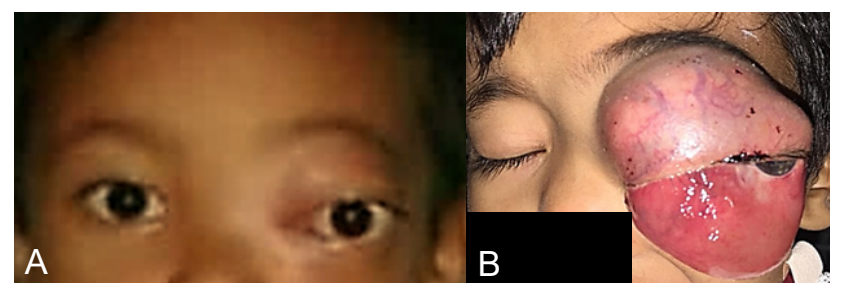

Figure 1. Clinical appearance. (A) when first realized proptosis (Source: Parent's photograph); (B) The first visit to the hospital.

Laboratory study found leukocytosis (16.840/uL) and anemia (9.1 gr\%). Blood hemostasis, liver function, renal function, and blood electrolytes were within normal limits. Chest X-ray examination showed no abnormalities in the heart and lung. Head Magnetic Resonance Imaging (MRI) showed a solid mass with the necrotic area, partially undefined borders, irregular edges, exophytic with the most extended size of $12 \times 6.6 \times 10.2 \mathrm{~cm}$, filling the left orbital cavity with varying density which showed enhancing solid components with in imaging with contrast. Conclusion on MRI examination was malignant soft tissue mass in the left orbital region with intracranial infiltration causing a midline shift of $1.7 \mathrm{~cm}$ (Figure 4).

The patient was planned to undergo tumor debulking under general anesthesia, and then the histopathological examination will be carried out to determine definitive management. The patient was given neoadjuvant chemotherapy at the third week of treatment using an extraocular retinoblastoma protocol of cyclophosphamide doxorubicin, vincristine, cytosine arabinoside and methotrexate, considering of the large size tumor and the risk of bleeding during surgery. This protocol was chosen because it was the most common case in our center. In addition, during the preparation for surgery, the patient was given supportive treatment and transfusion according to the condition.

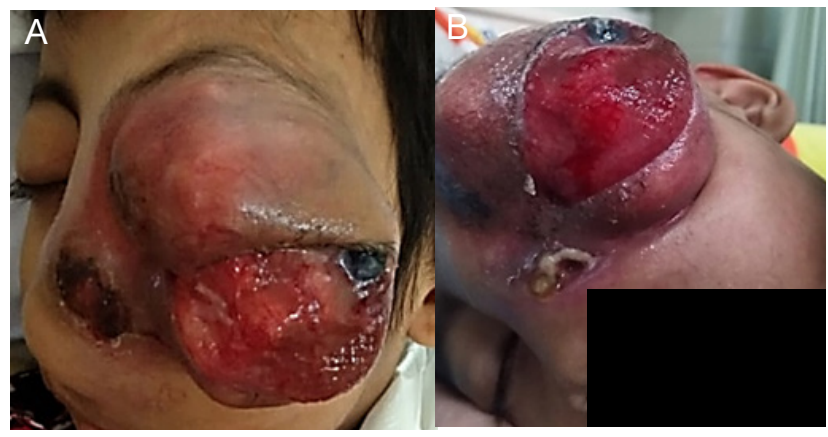

Figure 2. Clinical appearance after treatment with adjuvant chemotherapy; (A) The $4^{\text {th }}$ week of treatment, post a cycle of chemotherapy; (B) The $6^{\text {th }}$ week of treatment, post three cycles of chemotherapy.

In the fourth week of treatment, after giving the first cycle of adjuvant chemotherapy, the mass in the left eye did not develop, and the signs of inflammation were reduced (Figure 2); however, the patient's general condition was deteriorating. Unfortunately, there were no orbital radiotherapy facilities at our center, so the treatment options were limited. After giving adjuvant chemotherapy for three cycles in the sixth week, the patient's hemodynamic condition worsened. The patient had sepsis and was treated in the intensive care unit; however, two days later the patient could not survive.

\section{Discussion and conclusions}

The disease process involving orbit in pediatrics was substantially different from that found in adults. Proptosis was often the first sign of an orbital mass in a child. Most orbital lesions in children were benign, and few cases cause visual disturbances. However, in rare cases, orbital masses are malignant, rapidly growing, and life-threatening. Therefore, making a correct and prompt diagnosis was very important for the appropriate management and maintaining life and even visual function. ${ }^{[2]}$

The clinical features and radiological study of this patient seem to be consistent with orbital rhabdomyosarcoma; however, histopathology examination to establish the definitive diagnostic was required to exclude differential diagnoses such as neuroblastoma and glioma, also common in pediatrics. Neuroblastoma is a neuroendocrine tumor originating from the sympathetic nervous system in various regions. Orbital metastases often present as proptosis with periorbital ecchymoses. Radiologic findings include bone thickening, periosteal reaction (speculated bones), and lytic defects. On MRI ${ }^{[3]}$, neuroblastoma usually shows heterogeneous low signal 
intensity on T1-weighted images and high signal intensity on T2-weighted images. At the same time, glioma is a low-grade neoplasm that affects the precordial visual pathways of the optic nerve. Diagnosis is usually based on clinical examination and neuroimaging. Glioma usually appears as an isodense fusiform enlargement of the optic nerve with CT imaging. ${ }^{[4],[5]}$ The malignant tumor generally were characterized by an irregular shape, molding around orbital structures, diffuse and bony erosion ${ }^{[6]}$, which was similarly found in the patient.
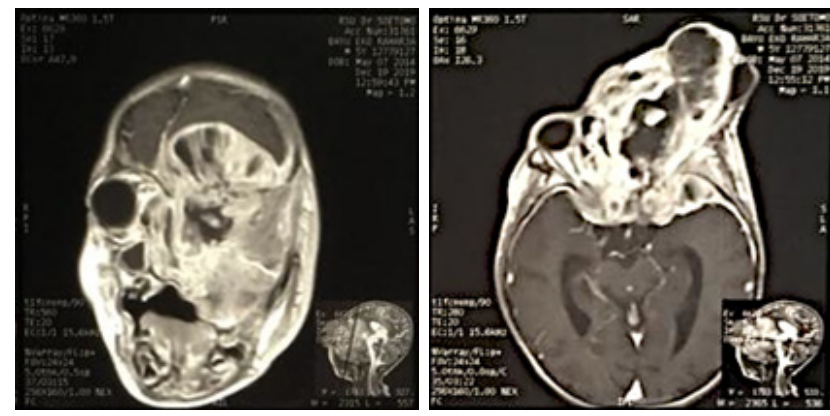

Figure 3. Computerised Tomography (CT) scan imaging.
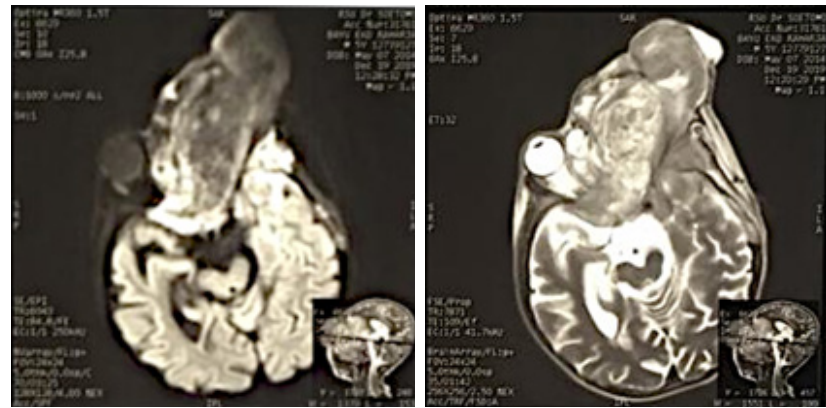

Figure 4. Magnetic Resonance Imaging (MRI); T1 and T2.

Retinoblastoma can resemble rhabdo-myosarcoma with a proptosis presentation in advance cases. ${ }^{[7]}$ The initial symptom of retinoblastoma was leukocoria, and the light comes from the white reflection of the tumor, typically red from the retina, giving a cat-eye appearance that could not be found on this patient. Invasion of the choroid, sclera to orbital extension can occur in the advanced phase; however, it always begins with intraocular spread. On the MRI scan of retinoblastoma, the mass was hyperintense in the vitreous at $\mathrm{T} 1$, hypointense at $\mathrm{T} 2$, with enhancement on T1 post-contrast. ${ }^{[1]}$ In this case, MRI has confirmed that there was no intraocular mass; therefore, the diagnosis of retinoblastoma seems to be ruled out.

Orbital rhabdomyosarcoma seems to be close to the diagnosis with available patient data. Orbital rhabdomyosarcoma generally presents with proptosis (80-100\%) that progresses quickly within a few weeks or globe displacement (80\%) to inferotemporal direction because primarily located superonasal. Orbital rhabdomyosarcoma can damage the orbital bone and infiltrate intracranially. The mass was generally extraconal (37-87\%) or extra and intraconal at once (13-
47\%), located close to the extraocular muscles with no muscle enlargement. In the early stages, the tumor is well-circumscribed; however, in later stages, the margin was irregular due to pseudo-capsular invasion. On T1 MRI, extraocular muscles showed isointense while the orbital fat hypointense, and on T2, both the extraocular muscles and orbital fat were hyperintense. ${ }^{[8]}$ The globe may be displaced or distorted; however, was rarely invaded. We also found these features in the patient.

The biopsy was a necessary action for diagnosis and determining prognosis. It can be done incisional or excisional depending on clinical conditions and imaging. Fine needle aspiration biopsy has limited tissue obtained for pathological diagnosis, so it is less valuable. Incision biopsy was preferred over excision to prevent the spread of the tumor to healthy tissue. The pathological diagnosis is established by the type of rhabdomyosarcoma: pleomorphic, embryonal, or alveolar. In pediatrics, most of the type was embryonal type. ${ }^{[1],[9]}$

Therapy given to the orbital tumors patients should be based on histopathological study and then treated according to the diagnostic protocol. Incisional biopsy was sufficient to be done because clear tumor margins are difficult to obtain. In addition, the prognosis was considered good after continued chemotherapy and radiation, regardless of the amount of tissue excised. [10] Suppose a diagnosis of rhabdomyosarcoma has been established, treatment can be given based on the Intergroup Rhabdomyosarcoma Study (IRS) by postsurgical staging system using a combination of intensive chemotherapy and radiotherapy. ${ }^{[8]}$

Pathological diagnosis assisting the selection of appropriate management. A report on the management of orbital tumors in children that were diagnosed as rhabdomyosarcoma showed satisfactory results using a protocol that included chemotherapy followed by radiotherapy and finally surgery. After 18 months of follow-up, there was no visual impairment and no tumor manifestations in other regions. The report states that a definitive diagnosis could be made based on tissue biopsies associated with histopathological and immunohistochemical studies. It was not easy to do if a surgical procedure for taking tissue samples was not possible. ${ }^{[11]}$

Unfortunately, in this case, due to a lack of knowledge of the patient's family, he came with a progressive condition. Besides, no radiotherapy facility has therapeutic potential, making a biopsy for histopathological examination very challenging. A wide variety of orbital neoplasms in pediatrics can present with a variety of clinical manifestations. Because the complexity of the site and the possibility of morbidity associated with biopsies in this region, a systemic and multidisciplinary 
approach were needed to facilitate an accurate diagnosis based on clinical or imaging examination. However, the general condition and hemodynamics of the patient must be prepared for surgery. Risks and complications during surgery may worse the condition, including massive bleeding and death on the operating table. ${ }^{[12]}$

The definitive diagnosis for the orbital tumor is obtained by histopathological examination. The investigation with CT scan or MRI imaging can be done if histopathological examination is not possible. Since the definitive diagnosis is still not assessed, the management can be affected. Chemotherapy and radiotherapy have shown a positive response mainly when they are carried out based on a proper pathological diagnosis. It is essential to be able to have a definitive diagnosis to provide adequate treatment for patients. Delayed case management makes orbital tumor become worse within a few weeks and potentially life-threatening.

\section{References}

[1] Lederman H, Rodrigues M, Tostes V, Caran E, Camargo M, Silva $\mathrm{F}$, et al. Differential diagnosis of orbital tumors in children. Int J Radiol Radiat Ther 2017;3:252-256. https:// doi.org/10.15406/ijrrt.2017.03.00068.

[2] Eghtedari M, Farsiani AR, Bordbar MR. Congenital orbital rhabdomyosarcoma. Ocul Oncol Pathol 2018;4:165-169. https://doi.org/10.1159/000481533.

[3] Yang W-J, Zhou Y-Y, Zhao F, Mei Z-M, Li S, Xiang Y. Orbital neuroblastoma metastasis: A case report and literature review. Medicine (Baltimore) 2019;98:e17038. https://doi. org/10.1097/md.0000000000017038.

[4] Rasool N, Odel JG, Kazim M. Optic pathway glioma of childhood. Curr Opin Ophthalmol 2017;28:289-295. https://doi.org/10.1097/icu.0000000000000370.

[5] Farazdaghi MK, Katowitz WR, Avery RA. Current treatment of optic nerve gliomas. Curr Opin Ophthalmol 2019;30:356363. https://doi.org/10.1097/icu.0000000000000587.

[6] Mombaerts I, Ramberg I, Coupland SE, Heegaard S. Diagnosis of orbital mass lesions: Clinical, radiological, and pathological recommendations. Surv Ophthalmol 2019;64:741-756. https://doi.org/10.1016/j.survophthal.2019.06.006.

[7] Chawla B, Pujari A, Behera AK. Extraocular retinoblastoma: A neglected case. BMJ Case Rep 2017;2017:bcr2016218471. https://doi.org/10.1136/bcr-2016-218471.

[8] Bradley JA, Kayton ML, Chi Y-Y, Hawkins DS, Tian J, Breneman J, et al. Treatment approach and outcomes in infants with localized rhabdomyosarcoma: A report from the soft tissue Sarcoma Committee of the Children's Oncology Group. Int J Radiat Oncol Biol Phys 2019;103:1927. https://doi.org/10.1016/j.jirobp.2018.08.017.

[9] Meel R, Singh P. Pediatric orbital tumors-An overview. CLEVER Clin Exp Vis Eye Res 2018;1:38.
[10] Thien HH, Kim Hoa NT, Duy PC, Carlos R-G, Son NH. Pediatric primary orbital rhabdomyosarcoma. J Pediatr Surg Case Reports 2020;59:101475. https://doi.org/10.1016/j. epsc.2020.101475.

[11] de Melo ACR, Lyra TC, Ribeiro ILA, da Paz AR, Bonan PRF, de Castro RD, et al. Embryonal rhabdomyosarcoma in the maxillary sinus with orbital involvement in a pediatric patient: Case report. World J Clin Cases 2017;5:440-445. https://doi. org/10.12998/wjcc.v5.i12.440.

[12] Kobayashi K, Matsumoto F, Miyakita Y, Arikawa M, Omura G, Matsumura S, et al. Risk factors for delayed surgical recovery and massive bleeding in skull base surgery. Biomed Hub 2020;5:1-14. https://doi.org/10.1159/000507750. 